\title{
Detection of OqxAB Efflux Pumps, a Multidrug-Resistant Agent in Bacterial Infection in Patients Referring to Teaching Hospitals in Ahvaz, Southwest of Iran
}

\author{
Mojtaba Moosavian $\left(\mathbb{D},{ }^{1,2}\right.$ Mahtab Khoshkholgh Sima, ${ }^{2}$ Nazanin Ahmad Khosravi, \\ and Effat Abbasi Montazeri ${ }^{2}$ \\ ${ }^{1}$ Infectious and Tropical Diseases Research Center, Health Research Institute, Ahvaz Jundishapur University of Medical Sciences, \\ Ahvaz, Iran \\ ${ }^{2}$ Department of Microbiology, School of Medicine, Ahvaz Jundishapur University of Medical Sciences, Ahvaz, Iran
}

Correspondence should be addressed to Mojtaba Moosavian; moosavian_m@yahoo.com

Received 3 June 2021; Revised 31 October 2021; Accepted 8 November 2021; Published 22 November 2021

Academic Editor: Ahmed Majeed Al-Shammari

Copyright $\odot 2021$ Mojtaba Moosavian et al. This is an open access article distributed under the Creative Commons Attribution License, which permits unrestricted use, distribution, and reproduction in any medium, provided the original work is properly cited.

\begin{abstract}
Antibiotic resistance mechanisms in Enterobacteriaceae are causative agents of global health problems. Bacterial infections due to multidrug resistance (MDR) may be mediated by the overexpression of efflux pumps. In this study, we investigated the prevalence of o $q x A$ and $\mathrm{o} q x B$ genes as two encoding agents of efflux pumps and the determination of antibiotic resistance rate in clinical isolates of Enterobacteriaceae. In this study, 100 Enterobacteriaceae isolates collected from different clinical specimens of infectious patients, such as wounds, urine, blood, discharge, and abscesses except stool, were examined. Identification of the isolates was performed using standard biochemical tests such as TSI, citrate, urea, lysine, SIM, MR-VP, and gas production. The antimicrobial susceptibility test was carried out by the Kirby-Bauer disk diffusion method according to CLSI guidelines, and finally, the oqxA and $o q x B$ genes were detected by the PCR method. Among 100 Enterobacteriaceae isolates, Escherichia coli and Enterobacter gergoviae were the most common isolates with $71 \%$ and $20 \%$, respectively. Also, the lowest isolates belonged to Enterobacter cloacae (3\%) and Klebsiella pneumoniae (1\%). Out of 100 Enterobacteriaceae isolates, 37 isolates (37\%) were positive for at least one of oq $x A$ or $o q x B$ genes, while both of these genes were detected among $12 \%$ of them. oq $x A B$ genes were detected in 8 cases of 20 (40\%) Enterobacter gergoviae and 4 cases of 71 (5.7\%) E. coli isolates. The antimicrobial susceptibility test showed that all isolates $(100 \%)$ were susceptible to imipenem, while the maximum resistance to piperacillin, ceftriaxone, and cefotaxime were $69 \%, 55 \%$, and $55 \%$, respectively. Also, the results of this study showed that antibiotic resistance in Enterobacteriaceae isolates caused by oqxAB genes is increasing among patients in Iran. Therefore, identification of resistant isolates and antibiotic monitoring programs are essential to prevent the spread of MDR isolates.
\end{abstract}

\section{Introduction}

Bacterial multidrug resistance (MDR) is an increasing problem in healthcare in both the hospital and community settings [1]. Gram-negative bacilli (GNB) resistance is a challenge for intensive care unit (ICU) physicians. GNB causes $45-70 \%$ of ventilator-associated pneumonia (VAP), $20-30 \%$ of catheter-related sepsis, and other ICU-acquired sepsis [2]. Mechanisms dependent on changes in membrane permeation processes has been reported as cause contributors of MDR [3]. At present time, it has been known that the plasmid-encoded OqxA and OqxB pumps confer resistance to multiple antimicrobial agents, such as quinolones (example, nalidixic acid) and fluoroquinolones (example, ciprofloxacin, norfloxacin, and flumequine) as well as biocides such as triclosan and chlorhexidine $[4,5]$.

The extended-spectrum cephalosporins and fluoroquinolones have been known as the choice drugs for the treatment of acute gastroenteritis caused by enteric pathogens. It has been now cleared that the resistance of these 
organisms to antimicrobials such as fluoroquinolone and quinolone could be due to mutation in the target region of quinolone resistance determining in DNA gyrases and type IV topoisomerases which ultimately prevent drugs from binding to this target [6].

Also, some of the reports show the multidrug efflux mechanisms that are widely conserved in bacteria are almost encoded by chromosomes. On the other hand, specific drug efflux mechanisms are usually encoded by plasmids and/or other mobile genetic elements (transposons and integrons) that carry resistance genes [7]. Pumps may transport a wide range of unlike compounds (including several classes of antibiotics). Such pumps can be associated with MDR [8].

The OqxAB efflux pump, a plasmid-mediated quinolone resistance (PMQR) element, has become widespread among members of Enterobacteriaceae over the past decade [9]. OqxAB pumps are encoded by two oq $x A$ and $o q x B$ genes, which are localized in one operon. The OqxAB efflux pumps cause resistance to fluoroquinolones (FQ) and other agents [10].

However, antibiotic resistance among some clinical isolates of Enterobacteriaceae such as E. coli, Klebsiella pneumoniae, and Salmonella are important not only in humans but also among animals, such as horses, swine, pork, and pigs, because some resistant agents such as the OqxAB pump encoded by plasmids (as pOLA 52), which harbored in these animals [6].

In this study, we investigated the prevalence of oq $x A$ and oq $x B$ genes and the determination of antibiotic resistance rate in Enterobacteriaceae isolates collected from clinical specimens of infectious patients.

\section{Materials and Methods}

2.1. Ethics Consideration. Ethics approval and consent to participate are not applicable in this study.

2.2. Bacterial Isolates from Specimens. In this study, 100 Enterobacteriaceae isolates from clinical specimens (wound, urine, blood, discharge, and abscesses except stool) which were collected by hospital laboratories from infectious patients were examined. These patients were referred to Golestan and Imam Khomeini Teaching Hospitals related to Ahvaz Jundishapur University of Medical Sciences, Ahvaz, Iran. After reculture on blood agar and MacConkey agar media, the isolates were identified by standard biochemical tests such as triple sugar iron agar (TSI), citrate, urea, lysine, SIM, MR-VP, and gas production (all culture media were provided by Merck, Germany) [11].

2.3. Antimicrobial Susceptibility Test. All isolates were subjected to antimicrobial sensitivity testing by the Kirby-Bauer disk diffusion method on Mueller-Hinton agar (MHA) (Merck, Germany) according to the Clinical and Laboratory Standard Institute (CLSI-2015) guidelines [12]. The tested antimicrobial agents were as follows: imipenem $(10 \mu \mathrm{g})$, ceftriaxone $(30 \mu \mathrm{g})$, ceftazidime $(30 \mu \mathrm{g})$, piperacillin $(100 \mu \mathrm{g})$, ciprofloxacin $(5 \mu \mathrm{g})$, gentamicin $(10 \mu \mathrm{g})$, amikacin $(30 \mu \mathrm{g})$, cefotaxime $(30 \mu \mathrm{g})$, norfloxacin $(10 \mu \mathrm{g})$, nalidixic acid $(30 \mu \mathrm{g})$, and levofloxacin $(5 \mu \mathrm{g})$. The antibiotic disks provided from MAST Group Ltd., Merseyside, U.K.

2.4. DNA Extraction and PCR of the oqxA and oqxB Genes. DNA template was prepared by the boiling method [13]. Briefly, 3-4 bacterial colonies were suspended in $500 \mu \mathrm{l} \mathrm{TE}$ buffer. The samples were incubated at $95^{\circ} \mathrm{C}$ for 15 minutes. Then, they were centrifuged for 10 minutes at $4^{\circ} \mathrm{C}$ and $12000 \mathrm{rpm}$, and the supernatants were stored in Eppendorf microtubes at $-20^{\circ} \mathrm{C}$, which were used as DNA templates. The concentration of the extracted DNA was measured by a photometer (Eppendorf, Germany) in 260/280 nm UV long waves. PCR master mixture was prepared in each $25 \mu \mathrm{l}$ reaction containing $2.5 \mu \mathrm{l}$ in 10X PCR buffer, $0.5 \mu \mathrm{l}$ of dNTP $\operatorname{mix}(10 \mathrm{mM}), 0.75 \mu \mathrm{lgCl}_{2}(50 \mathrm{mM}), 1 \mu \mathrm{l}$ of each primer (10 pmol) TAG, A/S Denmark (Table 1), $0.25 \mu \mathrm{l}$ of Taq DNA polymerase $(5 \mathrm{U} / \mu \mathrm{l}), 1 \mu \mathrm{l}$ of DNA template, and $18 \mu \mathrm{l}$ of distilled water.

DNA amplification of the oq $x A$ gene was performed in a thermocycler (Eppendorf, Germany) under initial denaturation at $94^{\circ} \mathrm{C}$ for $5 \mathrm{~min}$, followed by 34 cycles of denaturation at $94^{\circ} \mathrm{C}$ for $45 \mathrm{~s}$, annealing at $51^{\circ} \mathrm{C}$ for $45 \mathrm{~s}$, extension at $68^{\circ} \mathrm{C}$ for $1 \mathrm{~min}$, and the final step of extension at $72^{\circ} \mathrm{C}$ for $10 \mathrm{~min}$. These conditions for the oqxB gene were $94^{\circ} \mathrm{C}$ for $45 \mathrm{~s}$ (denaturation) followed by 32 cycles, $64^{\circ} \mathrm{C}$ for $45 \mathrm{~s}$, and $72^{\circ} \mathrm{C}$ for $60 \mathrm{~s}[14]$.

2.5. Electrophoresis. The PCR product was electrophoresed on 1.5\% agarose gel (Cinna GenCo, Iran) in 1X buffer Tris/ borate/EDTA buffer (Cinna GenCo, Iran) at $120 \mathrm{~V}$ for 60 minutes. The DNA was stained with ethidium bromide (Cinna GenCo, Iran), and photography of DNA amplified was performed in gel documentation (Viber Company, France). In this study, Klebsiella pneumoniae ATCC 700603 [4] and E. coli ATCC 25922 [14] were used as the positive control and negative control, respectively.

\section{Statistical Analysis}

To analyze the data, a chi-squared test was run in SPSS version 16 (SPSS Inc., Chicago, IL, USA). $P$ value $<0.05$ was considered statistically significant.

\section{Results}

Out of 100 Enterobacteriaceae isolates, the most isolates belonged to Escherichia coli $(71 \%)$, E. gergoviae (20\%), E. cloacae (3\%), E. aerogenes (5\%), and K. pneumoniae (1\%). The highest resistance was observed for piperacillin (69\%), ceftriaxone $(55 \%)$, cefotaxime $(55 \%)$, ceftizoxime $(43 \%)$, ceftazidime (42\%), nalidixic acid (39\%), ciprofloxacin (36\%), norfloxacin and gentamicin (26\%), and levofloxacin (23\%). The lowest resistance was observed for imipenem $(0 \%)$ and amikacin (5\%). The resistance rates to antimicrobial agents are given in Table 2 .

PCR results showed that out of 100 Enterobacteriaceae isolates, 37 isolates were positive for at least one of both 
TABle 1: Primers used in this study.

\begin{tabular}{|c|c|c|c|}
\hline Genes & Primer sequences & Product size (bp) & References \\
\hline$o q x A$ & $\begin{array}{c}\text { oqxAF-5'-CTCGGCGCGATGATGCT-3' } \\
\text { oqxAR-5'-CCACTCTTCACGGGAGACGA-3' }\end{array}$ & 392 & \\
\hline$o q x B$ & $\begin{array}{l}\text { oqxBs-5' }- \text { TTCTCCCCCGGCGGGAAGTAC-3' } \\
\text { oqxBa2-5' -CTCGGCCATTTTGGCGCGTA-3' }\end{array}$ & 512 & 14 \\
\hline
\end{tabular}

TABLE 2: The results of the antibiogram test for Enterobacteriaceae isolates.

\begin{tabular}{|c|c|c|c|c|c|c|c|c|c|c|c|c|c|}
\hline \multirow[b]{2}{*}{ Bacteria } & \multirow[b]{2}{*}{ Number } & \multicolumn{12}{|c|}{ Resistant (\%) } \\
\hline & & IMP & $\mathrm{CRO}$ & CZX & GM & $\mathrm{CP}$ & PIP & $\mathrm{AN}$ & CAZ & CTX & NA & NOR & LEV \\
\hline E. coli & 71 & 0 & 39 & 26 & 18 & 27 & 52 & 1 & 28 & 38 & 33 & 21 & 20 \\
\hline Enterobacter gergoviae & 20 & 0 & 9 & 10 & 6 & 9 & 12 & 4 & 9 & 14 & 6 & 5 & 3 \\
\hline Enterobacter cloacae & 3 & 0 & 3 & 2 & 1 & 0 & 2 & 0 & 3 & 1 & 0 & 0 & 0 \\
\hline Enterobacter aerogenes & 5 & 0 & 4 & 5 & 1 & 0 & 3 & 0 & 2 & 2 & 0 & 0 & 0 \\
\hline Klebsiella pneumoniae & 1 & 0 & 0 & 0 & 0 & 0 & 0 & 0 & 0 & 0 & 0 & 0 & 0 \\
\hline Total & 100 & 0 & 55 & 43 & 26 & 36 & 69 & 5 & 42 & 55 & 39 & 26 & 23 \\
\hline
\end{tabular}

IMP, imipenem; CRO, ceftriaxone; CZX, ceftizoxime; GM, gentamicin; CP, ciprofloxacin; PIP, piperacillin; AN, amikacin; CAZ, ceftazidime; CTX, cefotaxime; NA, nalidixic acid; NOR, norfloxacin; LEV, levofloxacin.

genes. Indeed, the prevalence of $o q x A$ and $o q x B$ genes among Enterobacteriaceae isolates was $22 \%$ and $15 \%$, respectively (Figures 1 and 2), while both oq $x A$ and oq $x B$ genes were found in $12 \%$ of them $(P>0.05)$. These results showed that all of positive results of efflux pump were related to the ciprofloxacin- and norfloxacine-resistant strains, and also, all of the susceptible isolates to the ciprofloxacin and norfloxacine were negative for efflux pump genes. oq $x A B$ genes were detected in $40 \%$ ( 8 of 20) of E. gergoviae and 5.7\% (4 of 71) of E. coli isolates.

\section{Discussion}

Bacterial multidrug resistance may be mediated by the overexpression of efflux pumps [15]. In MDR bacteria, overexpression of efflux pumps leads to reduced drug sensitivity by decreasing the intracellular concentration of antibiotics [3]. Dissemination of oqxAB genes may pose a great risk to food safety and public health [16].

In this study, we detected oq $x A B$ genes in clinical isolates of Enterobacteriaceae. Since bacterial resistance to antibiotics is related to different agents, in this study, detection of efflux pump genes was performed by PCR on all isolates to determine whether their resistance to antibiotics, including ciprofloxacin and norfloxacine, depended on the presence of these genes or other agents. Analysis of PCR results showed that although $37 \%$ of the isolates were positive for at least one $o q x A$ or $o q x B$ gene, the prevalence of both $o q x A$ and $o q x B$ was not significantly different between Enterobacteriaceae isolates $(P>0.05)$. Also, isolates sensitive to antibiotics, including ciprofloxacin and norfloxacine, lacked the oqxAB pump, while resistance isolates without efflux pump may be affected by other factors, such as reduced antibiotic permeability, decreased accumulation of intracellular antibiotics, and inactivation of the drugs. The incidence of oq $x A B$ genes was $40 \%$ and $5.7 \%$ for E. gergoviae and E. coli, respectively. Yuan et al. (2012) showed that $o q x B$ and $o q x B$ genes were present in $6.6 \%$ of E. coli strains [17]. Kao et al. (2016) reported $o q x A B$ genes in $6.05 \%$ of $E$. coli isolates [18].

Kim et al. (2009) investigated the prevalence of plasmidencoded multidrug efflux pump in clinical isolates of Enterobacteriaceae. In their survey, $0.4 \%$ of $E$. coli isolates and $4.6 \%$ of E. cloacae isolates were positive for both oqxB and $o q x B$ genes [14]. Because these studies were performed at different times, therefore, it could be the reason for different results. Zhao et al. (2010) reported the oqxA gene in $30.3 \%$ of $E$. coli isolates which were collected from farmworkers [19]. In our study, all oq $x A B$-positive Enterobacteriaceae strains were resistant to piperacillin, ceftriaxone, cefotaxime, ceftizoxime, ciprofloxacin, and ceftazidime, while all of these isolates (100\%) were susceptible to imipenem.

As some reports have confirmed that OqxA and OqxB efflux pumps confer resistance to multiple antimicrobial agents $(4,5)$, therefore, in our study also, the presence of OqxAB efflux pumps in clinical isolates of Enterobacteriaceae containing one or both oq $x A$ and oq $x B$ genes has conferred resistance to multiple antimicrobial agents.

In this study, the highest resistance was observed for piperacillin, ceftriaxone, and cefotaxime ( $\geq 55 \%)$, while Tang et al. (2016) showed that Enterobacteriaceae clinical isolates (carbapenem-resistant) were more resistant to some antibiotics, for example, their resistance (nonsusceptible) to cefuroxime, ceftazidime, and ampicillin was $100 \%$ and to piperacillin was $88.5 \%$ (20). In contrast to the study of Tang et al. (2016), which showed that the lowest resistance of these isolates to imipenem and amikacin was 32 and 20.5, respectively [20], in our study, the lowest resistance rate for imipenem and amikacin was $0 \%$ and $5 \%$, respectively. However, Ye et al. (2018) showed that $83.3 \%$ of Enterobacteriaceae clinical isolates (carbapenem-resistant) were simultaneously resistant to imipenem, meropenem, and ertapenem [21]. 


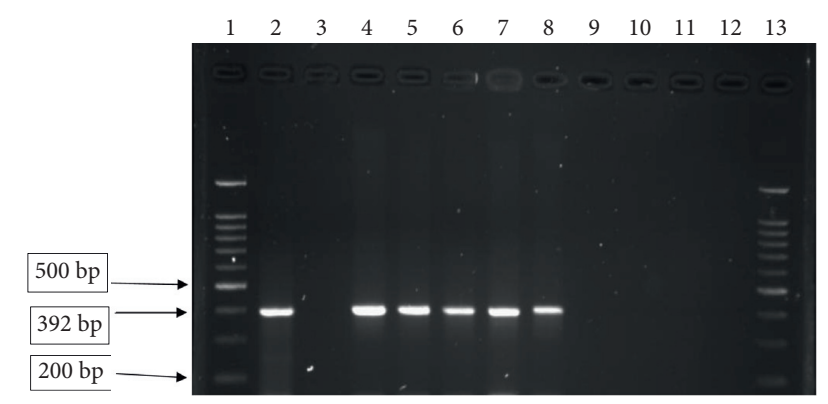

Figure 1: Electrophoresis of oq $x A$ gene PCR product. Lanes 1 and 13, 100 bp DNA ladder; lane 2, positive control (Klebsiella pneumoniae ATCC 700603); lane 3, negative control (E coli ATCC 25922); lanes $4-8$, positive samples for oq $x A$; lanes $9-12$, negative samples.

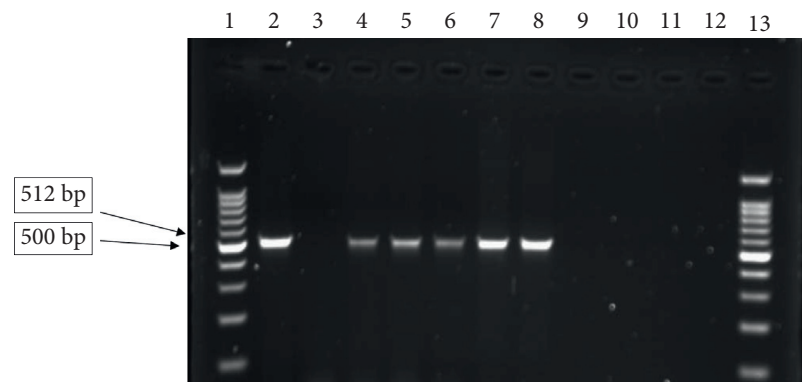

FIgURE 2: Electrophoresis of oq $x B$ gene PCR product. Lanes 1 and 13, 100 bp DNA ladder; lane 2, positive control (Klebsiella pneumoniae ATCC 700603); lane 3, negative control (E. coli ATCC 25922); lanes $4-8$, positive samples for $o q x B$; lanes $9-12$, negative samples.

Different resistance rates in various studies could be due to carrying two or more ESBLs, oq $x A B$, and carbapenemase genes or a combination of these genes in Enterobacteriaceae isolates.

In our study, the oqxA and $o q x B$ genes were not detected in $K$. pneumoniae, E. cloacae, and E. aerogenes isolates, whereas Park et al. (2012) showed oq $x A B$ genes in $14.4 \%$ of $K$. pneumoniae isolates, which were resistant to nalidixic acid, olaquindox, levofloxacin, and ciprofloxacin [5]. The results of our study also indicated that all oq $x A B$-positive E. coli strains and $75 \%$ of oq $x A B$-positive E. gergoviae strains were resistant to nalidixic acid. These results suggest that the presence of $o q x A B$ genes may be related to the resistance of these isolates to quinolone.

\section{Conclusion}

The results of this study showed that Enterobacteriaceae isolates with efflux pumps such as oq $x A B$ are increasing in Iran. Therefore, identification of resistant isolates and antibiotic monitoring programs are essential to prevent the spread of MDR isolates.

\section{Data Availability}

The data generated or analyzed during this study are included within this article.

\section{Additional Points}

Our study has the following limitations. For example, the different patterns of antibiotic resistance in each efflux pump and strain typing of isolates were not determined, while the presentation of them could help with more information for readers.

\section{Conflicts of Interest}

The authors declare that they have no conflicts of interest.

\section{Authors' Contributions}

Mojtaba Moosavian conceptualized the study, involved in project administration, and supervised and edited the article. Mahtab Khoshkholgh Sima developed the methodology and wrote and reviewed the article. Nazanin Ahmad Khosravi developed the methodology and validated and investigated the study. Effat Abbasi Montazeri visualized the study and analyzed the data.

\section{Acknowledgments}

This study was a part of the MSc thesis of Nazanin Ahmad Khosravi approved in Ahvaz Jundishapur University of Medical Sciences (No. B-9101), Ahvaz, Iran. The authors' appreciation goes to Vice of Chancellor for Research Affairs, and Tropical and Infectious Diseases Research Center, Ahvaz Jundishapur University of Medical Sciences, Ahvaz, Iran, for their financial and executive support.

\section{References}

[1] R. A. Weinstein and D. C. Hooper, "Efflux pumps and nosocomial antibiotic resistance: a primer for hospital epidemiologists," Clinical Infectious Diseases, vol. 40, no. 12, pp. 1811-1817, 2005.

[2] É Ruppé, P. L. Woerther, and F. Barbier, "Mechanisms of antimicrobial resistance in Gram-negative bacilli," Annals of Intensive Care, vol. 5, p. 61, 2015.

[3] H. Nikaido and J.-M. Pagès, "Broad-specificity efflux pumps and their role in multidrug resistance of Gram-negative bacteria," FEMS Microbiology Reviews, vol. 36, no. 2, pp. 340-363, 2012.

[4] J. M. Rodríguez-Martínez, P. Díaz de Alba, A. Briales et al., "Contribution of oqxAB efflux pumps to quinolone resistance in extended-spectrum- $\beta$-lactamase-producing Klebsiella pneumoniae," Journal of Antimicrobial Chemotherapy, vol. 68, pp. 68-73, 2013.

[5] K. S. Park, M. H. Kim, T. S. Park, Y. S. Nam, H. J. Lee, and J. T. Suh, "Prevalence of the plasmid-mediated quinolone resistance genes, aac $\left(6^{\prime}\right)$-Ib-cr, qepA, and oqxAB in clinical isolates of extended-spectrum $\beta$-lactamase (ESBL)-producing Escherichia coli and Klebsiella pneumoniae in Korea," Annals of Clinical Laboratory Science, vol. 42, pp. 191-197, 2012.

[6] M. H. Wong, E. W. Chan, L. Z. Liu, and S. Chen, "PMQR genes oqx $\mathrm{AB}$ and aac $\left(6 \hat{a} €^{2}\right) \mathrm{Ib}-\mathrm{cr}$ accelerate the development of fluoroquinolone resistance in Salmonella typhimurium," Frontiers in Microbiology, vol. 5, p. 521, 2014.

[7] K. Poole, "Efflux-mediated antimicrobial resistance," Journal of Antimicrobial Chemotherapy, vol. 56, no. 1, pp. 20-51, 2005. 
[8] M. A. Webber and L. Piddock, "The importance of efflux pumps in bacterial antibiotic resistance," Journal of Antimicrobial Chemotherapy, vol. 51, no. 1, pp. 9-11, 2003.

[9] M. H. Y. Wong, E. W. C. Chan, and S. Chen, "Evolution and dissemination of OqxAB-like efflux pumps, an emerging quinolone resistance determinant among members of Enterobacteriaceae," Antimicrobial Agents and Chemotherapy, vol. 59, no. 6, pp. 3290-3297, 2015.

[10] N. Kulková, M. Babálová, J. Brnová, and V. Krcméry, "Transferable fluoroquinolone resistance in Enterobacteriaceae and Pseudomonas aeruginosa isolated from hemocultures," Central European Journal of Public Health, vol. 22, pp. 60-63, 2014.

[11] P. M. Tille, Diagnostic Microbiologypp. 308-328, Elsevier, St.Louis, MI, USA, 13th edition, 2014.

[12] CLSI, Performance Standards for Antimicrobial Susceptibility Testing: Twenty-Fifth Informational Supplement, Clinical \& Laboratory Standards Institute, Wayne, IL, USA, 2015.

[13] M. Safari, M. Shojapour, M. Akbari, A. Pourbabaee, and H. Abtahi, "Dissemination of CTX-M-type beta-lactamase among clinical isolates of Enterobacteriaceae in Markazi province, Iran," Jundishapur Journal of Microbiology, vol. 6, p. e7182, 2013.

[14] H. B. Kim, M. Wang, C. H. Park, E.-C. Kim, G. A. Jacoby, and D. C. Hooper, "OqxAB encoding a multidrug efflux pump in human clinical isolates of Enterobacteriaceae," Antimicrobial Agents and Chemotherapy, vol. 53, no. 8, pp. 3582-3584, 2009.

[15] M. Martins, M. Viveiros, I. Couto et al., "Identification of efflux pump-mediated multidrug-resistant bacteria by the ethidium bromide-agar cartwheel method," In vivo, vol. 25, pp. 171-178, 2011.

[16] J. Li, H. Zhang, J. Ning et al., "The nature and epidemiology of OqxAB, a multidrug efflux pump," Antimicrobial Resistance and Infection Control, vol. 8, no. 1, p. 44, 2019.

[17] J. Yuan, X. Xu, Q. Guo et al., "Prevalence of the oqxAB gene complex in Klebsiella pneumoniae and Escherichia coli clinical isolates," Journal of Antimicrobial Chemotherapy, vol. 67, no. 7, pp. 1655-1659, 2012.

[18] C.-Y. Kao, H.-M. Wu, W.-H. Lin et al., "Plasmid-mediated quinolone resistance determinants in quinolone-resistant Escherichia coli isolated from patients with bacteremia in a university hospital in Taiwan, 2001-2015," Scientific Reports, vol. 6, no. 1, p. 32281, 2016.

[19] J. Zhao, Z. Chen, S. Chen et al., "Prevalence and dissemination of oqxAB in Escherichia coli isolates from animals, farmworkers, and the environment," Antimicrobial Agents and Chemotherapy, vol. 54, no. 10, pp. 4219-4224, 2010.

[20] H.-J. Tang, C.-F. Hsieh, P.-C. Chang et al., "Clinical significance of community- and healthcare-acquired carbapenemresistant Enterobacteriaceae isolates," PLoS One, vol. 11, no. 3, p. e0151897, 2016.

[21] Y. Ye, L. Xu, Y. Han, Z. Chen, C. Liu, and L. Ming, "Mechanism for carbapenem resistance of clinical Enterobacteriaceae isolates," Experimental and therapeutic medicine, vol. 15, pp. 1143-1149, 2018. 